Published in final edited form as:

Nat Chem. 2018 December ; 10(12): 1213-1221. doi:10.1038/s41557-018-0147-z.

\title{
Inhibiting Amyloid-ß cytotoxicity through its interaction with the cell surface receptor LilrB2 by structure-based design
}

\author{
Qin Cao ${ }^{1}$, Woo Shik Shin ${ }^{2}$, Henry Chan ${ }^{3}$, Celine K. Vuong ${ }^{4,5}$, Bethany Dubois ${ }^{2,6}$, Binsen \\ Li $^{2,3}$, Michael R. Sawaya ${ }^{1}$, Juli Feigon ${ }^{3}$, Douglas L. Black ${ }^{4}$, David S. Eisenberg ${ }^{1,{ }^{*}}$, and Lin \\ Jiang ${ }^{2, *}$ \\ ${ }^{1}$ Departments of Chemistry and Biochemistry and Biological Chemistry, UCLA-DOE Institute, \\ and Howard Hughes Medical Institute, UCLA, Los Angeles CA 90095-1570 \\ 2 Department of Neurology, Molecular Biology Institute, and Brain Research Institute, UCLA, Los \\ Angeles CA 90095 \\ ${ }^{3}$ Department of Chemistry and Biochemistry, UCLA, Los Angeles CA 90095-1569 \\ ${ }^{4}$ Department of Microbiology, Immunology, and Molecular Genetics, David Geffen School of \\ Medicine, UCLA, Los Angeles CA 90095 \\ ${ }^{5}$ current address: Systems Neurobiology Laboratory, The Salk Institute for Biological Studies, \\ 10010 North Torrey Pines Road, La Jolla, CA 92037 \\ ${ }^{6}$ current address: Division of Applied Mathematics, Brown University, Providence, RI 02912
}

\begin{abstract}
Inhibiting the interaction between $\beta$-amyloid $(\mathrm{A} B)$ and a neuronal cell surface receptor, LilrB2, has been suggested as a potential route for treating Alzheimer's disease (AD). Supporting this approach, AD-like symptoms are reduced in mouse models following genetic depletion of the LilrB2 homolog. In its pathogenic, oligomeric state, $A ß$ binds to LilrB2, triggering a pathway to synaptic loss. Here we identified the LilrB2 binding moieties of $A ß\left({ }^{16} \mathrm{KLVFFA}^{21}\right)$ and identified its binding site on LilrB2 from a crystal structure of LilrB2 immunoglobulin domains D1D2 complexed to small molecules that mimic phenylalanine residues. In this structure, we observed two pockets that can accommodate the phenylalanine sidechains of KLVFFA. These pockets were confirmed to be ${ }^{16} \mathrm{KLVFFA}^{21}$ binding sites by mutagenesis. Rosetta docking revealed a plausible
\end{abstract}

Users may view, print, copy, and download text and data-mine the content in such documents, for the purposes of academic research, subject always to the full Conditions of use: http://www.nature.com/authors/editorial_policies/license.html\#terms

*Corresponding Author: David S. Eisenberg, david@mbi.ucla.edu; Lin Jiang, jianglin@ucla.edu. AUTHOR CONTRIBUTIONS

Q.C., D.S.E and L.J. conceived and designed the experiments. Q.C., W.S.S., H.C., C.K.V, B.D. and B.L. performed the experiments. W.S.S., B.D. and L.J. performed computational docking and structure-guided selection of small molecules. H.C. and J.F. performed and analyzed NMR experiments. B.L. and L.J. performed and analyzed CD experiments. C.K.V. and D.L.B. cultured primary neurons. Q.C. and M.R.S. solved the structure of LilrB2 and benzamidine complex. All authors discussed the results and commented on the manuscript. Q.C., D.S.E. and L.J. analyzed the data and co-wrote the paper.

Competing Financial Interests

DSE is an advisor and equity shareholder in ADDRx, Inc.

DATA AVAILABILITY

The crystal structure reported here, LilrB2 D1D2 complexed with benzamidine, and the corresponding diffraction data have been deposited to the Protein Data Bank (PDB) with the accession code 6BCS. All other data are available upon reasonable request to the authors. 
geometry for the Aß-LilrB2 complex and assisted with the structure-guided selection of small molecule inhibitors. These molecules inhibit $\mathrm{A} \beta$-LilrB2 interactions in vitro and on the cell surface and reduce $A ß$ cytotoxicity, which suggests these inhibitors are potential therapeutic leads against $\mathrm{AD}$.

\section{Graphical Abstract}

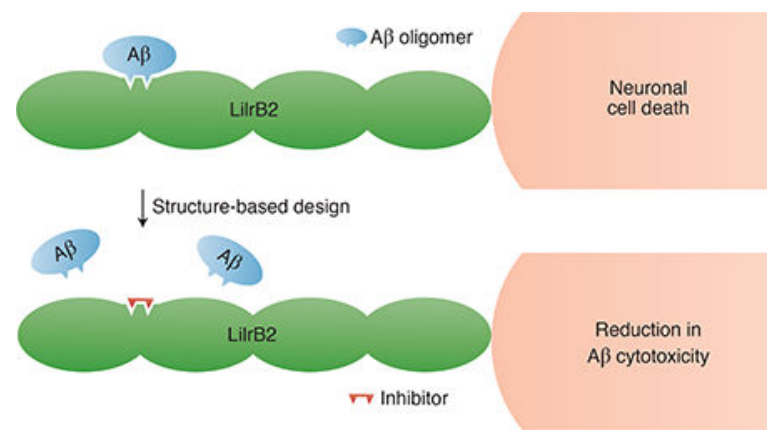

\section{Keywords}

Alzheimer's disease; neuronal cell surface receptor; structure-based design

\section{INTRODUCTION}

Aggregated $B$-amyloid $(A \beta)$ is found in large amounts in the autopsied brains of Alzheimer's Disease (AD) patients, and it is widely considered as a key factor in triggering neural degeneration in $\mathrm{AD}^{1,2}$. The longstanding amyloid cascade hypothesis has been challenged in recent years by the lack of correlation between $A ß$ accumulation and cognitive impairment in elderly patients and the better correlation between histopathological changes of neurofibrillary tangles (aggregation of tau) and loss of cognition ${ }^{3-5}$. However, three major findings continue to support the hypothesis: $A ß$ overproduction is found in nearly all familial forms of $\mathrm{AD}^{6,7}$, the oligomeric form of $\mathrm{A} \beta$ is toxic to neurons ${ }^{8-10}$, and overexpression of $\mathrm{A} \beta$ and APP (amyloid precursor protein) mutants in animal models leads to the development of $\mathrm{AD}$ related phenotypes ${ }^{11,12}$. Recent AD related studies continue to support the key role of $\mathrm{A} \beta^{13,14}$.

Extensive work has focused on developing inhibitors of $A B$ toxicity as potential therapeutic drugs for $\mathrm{AD}$. Most of these target $\mathrm{A} B$ aggregation (e.g. ${ }^{15-17}$ ), reduce the production of $\mathrm{A} B$ through inhibition of $B$ or $\gamma$-secretase (e.g. ${ }^{18-20}$ ), or reduce $A ß$ levels through immunotherapy ${ }^{21}$ (e.g. ${ }^{22}$ ). Recent studies suggest that one or more high-affinity protein receptors on the neuronal cell surface, such as cellular prion protein $\left(\operatorname{PrP}^{\mathrm{C}} 23\right)$ and ephrin type $\mathrm{B}$ receptor $2\left(\mathrm{EphB} 2{ }^{24}\right)$, are responsible for the recruitment of $\mathrm{A}$ oligomers and subsequent neurotoxicity ${ }^{25}$. These findings have sparked interest in illuminating the molecular mechanism of Aß-receptor recognition, with the hope that this information will lead to the development of new, effective $A D$ therapeutics that inhibit the interaction of $A B$ with neuron cell receptors. 
Among all these cell surface $A B$ receptors ${ }^{25}$, LilrB2 is one of a few receptors that are reported to be promising therapeutic targets for treatment of $\mathrm{AD}$, based on the observation that genetic depletion of the murine homolog, $\mathrm{PirB}\left(\mathrm{PirB}^{-/-}\right)$, rescues Aß induced ADrelated phenotypes in multiple model systems from cultured cortical neurons to transgenic mice, including recognition memory defects in APP/PS1 mice ${ }^{26}$. The two amino-terminal extracellular immunoglobulin domains (D1D2) of LilrB2 and its murine homolog PirB selectively bind $A ß$ oligomers with nanomolar affinity. LilrB2 protein is detected in human brains of both $\mathrm{AD}$ patients and non-AD adults, with no significant difference in expression level, but its downstream signaling is altered in AD brains, implicating LilrB2 in Aßdependent synaptic loss ${ }^{26}$. Here we identify the binding moieties of both Aß oligomers and LilrB2 and present a model for their interaction. Based on the structural model, we designed Aß-LilrB2 interaction inhibitors by computationally selecting molecules to compete with $A ß$ for the LilrB2 binding sites. The resulting compounds inhibited the interaction between oligomeric $\mathrm{A} ß$ and LilrB2 with up to high nanomolar $\mathrm{K}_{\mathrm{i}}$ and low micromolar $\mathrm{IC}_{50}$ values. They also showed the ability to inhibit LilrB2 induced Aß-cell contact, and therefore to inhibit Aß cytotoxicity.

\section{RESULTS}

\section{Mapping the core region of $A \beta$ binding to the LilrB2 D1D2 domains}

A 200-residue recombinant LilrB2 segment spanning the D1 and D2 domains (LilrB2 D1D2) was used in our study, and oligomeric human $A \beta_{1-42}$ (A342) was prepared by incubating $10 \mu \mathrm{M} \mathrm{AB42}$ at $37^{\circ} \mathrm{C}$ overnight (Supplementary Fig. 1). LilrB2 D1D2 selectively binds oligomeric Aß42 as previously reported ${ }^{26}$ (Supplementary Fig. 2). To map the binding core of $A \beta$, we developed an ELISA-based interaction assay that enables high-throughput detection of the Aß-LilrB2 interaction. We immobilized LilrB2 D1D2 on an ELISA plate and measured the amount of bound $A \beta$ segments by $A ß$ specific antibodies or the fluorescence signal of fluorescein conjugated to the segments. We found the LilrB2 D1D2 domains bind to $A \beta 42$ and its amino-terminal moiety $A \beta_{1-21}$, but not to $A \beta_{1-15}$ (Fig. 1 and Supplementary Table 1). We also found that the LilrB2 D1D2 domains bind to A $\beta_{15-35}$ but not $A \beta_{22-42}$ (Supplementary Fig. 2). These results indicate that the $A ß$ segment ${ }^{16} \mathrm{KLVFFA}^{21}$, which is also widely considered as a key element of $\mathrm{A} \beta$ aggregation $^{27,28}$, is the core region that binds to LilrB2 D1D2.

We then tested the interaction of LilrB2 D1D2 with ${ }^{16} \mathrm{KLVFFA}^{21}$ derived peptides. We did not detect binding of LilrB2 D1D2 to the peptide that contains a single copy of ${ }^{16} \mathrm{KLVFFA}^{21}$ (A $\beta_{16-21}$, Fig. 1). However, our experiment showed it did bind to a tandem repeat design of ${ }^{16}$ KLVFFA $^{21}$ (A $\beta_{16-21}$-TR, sequence KLVFFAPDGKLVFFA, Fig. 1, Supplementary Table 1). The binding we observed with the tandem repeats was not due to introduction of the ProAsp-Gly linker between the two ${ }^{16} \mathrm{KLVFFA}^{21}$ copies, since the control peptide with a single copy of ${ }^{16} \mathrm{KLVFFA}^{21}$ and the linker (A $\beta_{16-21}-\mathrm{C}$, sequence KLVFFAPDG) did not bind to LilrB2 D1D2 (Fig. 1). These results suggest that two copies of ${ }^{16} \mathrm{KLVFFA}^{21}$ represent a minimal $\mathrm{A} B$ oligomer and the core epitope for LilrB2 binding. Moreover, the observation that the tandem repeat but not the single copy of ${ }^{16} \mathrm{KLVFFA}^{21}$ binds to LilrB2 suggests that LilrB2 recognizes a particular conformation in addition to the primary amino acid sequence. 
We hypothesize that the antiparallel dimer of ${ }^{16} \mathrm{KLVFFA}^{21}$, rather than a single copy, readily assembles into a minimal oligomer, reasoning that the tandem linkage lowers the entropy barrier to oligomer formation (see discussion).

\section{Crystal structure of LilrB2 D1D2 complexed with benzamidine}

We mixed LilrB2 D1D2 with various Aß segments and screened for crystals, and we determined the crystal structure of LilrB2 D1D2 mixed with $\mathrm{A}_{14-23}$ at $2.1 \AA$ resolution (Supplementary Table 2). In this structure, no density for the $A B$ segment was found, which is consistent with no detectable binding of ${ }^{16} \mathrm{KLVFFA}^{21}$ monomer to LilrB2 D1D2. Instead we found four benzamidine (Ben) molecules (Fig. 2a) which were used as an additive for crystal optimization. The presence and positions of the benzamidine molecules were determined by inspection of difference electron density maps $\left(\mathrm{F}_{\mathrm{o}}-\mathrm{F}_{\mathrm{c}}\right.$, Supplementary Fig. 3) and the surrounding environment (Fig. $2 \mathrm{~b}$ and c). The chemical structure of benzamidine is similar to that of phenylalanine (Fig. 2a), so it mimics the binding of phenylalanine from the ${ }^{16} \mathrm{KLVFFA}^{21}$ binding core of Aß. We chose the binding pockets of Ben 3 and 4 for further investigation because of the following observations: First, the binding pockets of Ben 3 and 4 are close to each other (separated by $7.5 \AA$ ) and are both located in the groove between D1 and D2 domains (Fig. 2a). Given that at least two copies of ${ }^{16} \mathrm{KLVFFA}^{21}$ are required to bind to LilrB2 (Fig. 1) and each copy has two phenylalanines, it is likely that the binding sites for ${ }^{16} \mathrm{KLVFFA}^{21}$ on LilrB2 have two phenylalanine binding pockets close to each other. Second, most residues comprising the Ben 3 and 4 binding pockets are hydrophobic with geometry suitable for binding bulky hydrophobic residues such as phenylalanine (Ile ${ }^{154}$, $\mathrm{Tyr}^{199}$, Pro ${ }^{204}$, Tyr ${ }^{205}$ and $\operatorname{Trp}^{207}$ for Ben 3 and Val ${ }^{38}$, Cys ${ }^{156}$, Pro ${ }^{164}$, Cys ${ }^{166}$ and Trp ${ }^{207}$ for Ben 4) (Fig. 2a, b and c). Hydrogen bonding (Ben 3 with Gly ${ }^{51}$ and Asn ${ }^{168}$; Ben 4 with $\mathrm{Asp}^{36}$ ) and crystal lattice contacts (Ben 3 with $\mathrm{Gly}^{51}$ and Ben 4 with Leu ${ }^{53}$ ) also stabilize benzamidine binding, but are minor contributors and appear unnecessary for binding phenylalanine. Third, Asp ${ }^{36}$ and other negatively charged residues are located adjacent to the groove (Supplementary Fig. 3), close enough to neutralize the positive charge of Lys ${ }^{16}$ of ${ }^{16} \mathrm{KLVFFA}^{21}$ and further stabilize its binding. Fourth, by superimposing our complex on the ligand free LilrB2 D1D2 structure (PDB ID 2GW5 ${ }^{29}$ ), we found that upon ligand binding, the binding groove widens due to movement of $\beta$-strand of residues 165 to 168 . In addition, the loop composed of residues 159 to 164 , which is disordered and lacking electron density in the ligand-free structure, becomes ordered and forms a protective cap over the binding groove (Supplementary Fig. 3). These slight conformational changes make this groove a better binding site for both benzamidine and presumably the Aß binding core. On the basis of this structural analysis, we hypothesized that the binding pockets of Ben 3 and 4 on LilrB2 are the binding sites for ${ }^{16} \mathrm{KLVFFA}^{21}$ of $\mathrm{A} \beta$; in the following we provide support for this hypothesis by mutagenesis and Rosetta docking.

\section{Validation of the binding sites of LilrB2 by mutagenesis and Rosetta docking}

We designed three LilrB2 mutations to validate the putative binding sites for $A ß$. We first chose $\mathrm{Asn}^{168}$ and $\mathrm{Val}^{38}$, whose side chains participate in the Ben 3 and 4 pockets respectively (Fig. $2 \mathrm{~b} \& \mathrm{c}$ ). We mutated both to tryptophan to block these two pockets by creating steric hindrance with the ligands (Supplementary Fig. 3). We also designed the D36G mutation to target $\mathrm{Asp}^{36}$ that putatively neutralizes the negative charge of Lys ${ }^{16}$ of 
Aß. The three resulting mutant proteins, LilrB2 D1D2 D36G, V38W and N168W, bound significantly lower amounts of full length $A \beta$ as well as the ${ }^{16} \mathrm{KLVFFA}^{21}$ tandem repeat (Aß16-21-TR) compared to wildtype at the same loading concentration in ELISA-based interaction assays (Fig. 3a and Supplementary Fig. 4). Size exclusion chromatography shows that all three mutants elute at the same retention volume as wild type (Supplementary Fig. 4), and ${ }^{1} \mathrm{H}^{15} \mathrm{~N}-\mathrm{HSQC}$ spectra show that these mutants have similar chemical shift patterns as wildtype (Supplementary Fig. 4), which indicates that diminished strength of these interactions is not due to changes in overall folding or the aggregation state of LilrB2. These results indicate that blocking Ben 3 and 4 binding pockets by single mutations diminishes the binding of LilrB2 for both full length $A ß$ and the ${ }^{16} \mathrm{KLVFFA}^{21}$ tandem repeat. Therefore, these results support our hypothesis that Ben 3 and 4 binding pockets are the binding sites for ${ }^{16} \mathrm{KLVFFA}^{21}$ in the tandem repeat and in full length $\mathrm{A} B$.

To further validate the binding sites on LilrB2 and to develop a model of Aß-LilrB2 interaction, we applied Rosetta flexible peptide docking ${ }^{30}$ to dock the ${ }^{16} \mathrm{KLVFFA}^{21}$ segment to LilrB2 D1D2. We used our crystal structure shown in Fig. 2 as a starting model of LilrB2. An antiparallel $\beta$-sheet unit was taken from the crystal structure of the ${ }^{16} \mathrm{KLVFFA}^{21}$ steric zipper (PDB ID 3OW9 ${ }^{28}$ ), in order to represent a minimal $\beta$-sheet conformation of oligomeric $A \beta^{31}$, and the tandem repeat of $A \beta_{16-21}$-TR (see discussion). During docking simulations, we confined KLVFFA to contact three key residues $\left(\mathrm{Asp}^{36}, \mathrm{Val}^{38}, \mathrm{Asn}^{168}\right.$ ) that we identified as important for $A \beta$ binding in our mutagenesis experiments. To minimize the influence of the starting orientation of the peptide, we placed two strands of KLVFFA away from the groove between the D1 and D2 domains (putative binding site) in a random orientation. Notably, we imposed no restraints to occupy the putative binding pockets identified in our crystal structure with benzamidine. For each starting conformation, 50,000 models were generated and the top 500 models with favorable Rosetta energies were further refined by energy optimization. After refinement, the five models ranked by Rosetta energies $^{32}$ and shape complementary ${ }^{33}$ were selected for visual inspection. We found one model with two phenylalanine residues located within the binding pockets of Ben 3 and 4 (Fig. 3b to d). In this model, two independent KLVFFA molecules associated as an antiparallel B-sheet and docked in the groove between D1 and D2. Phe ${ }^{20}$ and Phe ${ }^{19}$ from separate molecules docked in the pockets of Ben 3 and 4, respectively. The RMSD of the aromatic rings between phenylalanine residues and benzamidine molecules are $2.3 \AA$. These docking results support our prediction of LilrB2 binding sites and provide a putative model of Aß-LilrB2 interaction. No other plausible $A ß$ conformation was generated by our computational docking that fits two phenylalanines in these putative LilrB2 binding sites.

\section{Structure-based design of $A \beta$-LilrB2 interaction inhibitors}

We designed AB-LilrB2 interaction inhibitors (ALI) to occupy the binding sites on LilrB2 and prevent $\mathrm{A} \beta$ binding, as directed by our structural model of the Aß-LilrB2 complex. Our approach, adapted from previous work ${ }^{34}$, combines knowledge of amyloid structures and computational screening to discover small molecules that interact with Aß fibrils and protect cells against their toxicity. We searched a compound library of $\sim 32,000$ small molecules, including approved drugs, drugs in animal tests and clinical trials, and natural products whose pharmacokinetic (PK) and/or toxicity profile is known (Supplementary Fig. 5 and 
Supplementary method). Small molecules that can potentially mimic the conformation of the aromatic rings of the ligand in our crystal structure were selected and docked to the binding pocket of LilrB2. The compounds were then ranked by their predicted binding energy and the similarity between the docked model and the crystal structure. Finally, 12 top-ranking small molecules (ALI 1-12) were chosen for experimental characterization based on their shape similarity, computational docking energy, and potential to cross the blood-brain barrier (Supplementary Table 3).

We tested the inhibitory efficiency of all candidates by quantitative immunoprecipitation assays with LilrB2 D1D2 and oligomeric Aß42. At a molar ratio of 1:10:50 (LilrB2:Aß:inhibitor), 9 out of 12 candidates show inhibition of Aß-LilrB2 interaction with a lower $A ß$ binding signal that considered to be statistically significant compared to the controls with no inhibitor added (Fig. 4a). Six candidates (ALI4, 5, 6, 7, 9 and 10) were selected for concentration dependent studies, and all of them inhibited Aß-LilrB2 interaction in a dose-dependent manner (Fig. 4b and Supplementary Fig. 6). Their docking models created by high resolution Rosetta docking are superimposed with benzamidine and are shown in Fig. 4c and Supplementary Fig. 6. The statistical analysis of the concentration dependent studies shows that all 6 candidates have high nanomolar to low micromolar $\mathrm{K}_{\mathrm{i}}$ and low micromolar $\mathrm{IC}_{50}$ values (Fig. $4 \mathrm{~d}$ ). These results suggest that structure-based design was successful in identifying small-molecule inhibitors that block Aß-LilrB2 interaction in vitro.

\section{Tests of inhibitors by cell-based assays}

To test the inhibitors on cells, we transiently transfected HEK293T cells with full-length LilrB2 having monomer red fluorescent protein (mRFP) conjugated at its carboxy-terminus (LilrB2-mRFP). We then tested the interaction of LilrB2 with exogenously added oligomeric Aß42 conjugated at its amino-terminus with fluorescein (FITC-Aß42, Supplementary Fig. 7). HEK293T cells transfected with mRFP alone were used as a negative control. After 3 hours of incubation with FITC-Aß42, we fixed and washed the cells, and found the cells transfected with mRFP bind only $13 \%$ of FITC-Aß42 relative to cells transfected with LilrB2-mRFP (Fig. 5a \& b), consistent with previously reported results using HEK293 cells expressing LilrB2 or PirB ${ }^{26}$. These results indicate LilrB2 induces Aß-cell interaction. When we added a $10 \mu \mathrm{M}$ concentration of our inhibitors to cells before adding FITC-Aß42, we found the amount of bound Aß42 was significantly reduced; the lowest values are 27\% (ALI10) and 29\% (ALI6) relative to the controls in which no inhibitor was added (Fig. 5a \& b). These results indicate our selected small molecules inhibit Aß-LilrB2 interaction at the cellular level, and therefore inhibit LilrB2 induced Aß-cell contact.

We selected candidate compound ALI6 to examine its effect on the cytotoxicity of AB, because ALI6 exhibits the best score in the computational docking and the best inhibitory activity both in vitro and on cell levels. We transfected HEK293T cells with LilrB2-mRFP and treated them with $500 \mathrm{nM}$ oligomeric Aß42 for 24 hours. Cell viability (MTT) assays showed that $38 \%$ of cells were killed relative to controls in which the cells were incubated with PBS buffer solutions (Fig. 5c). Further cell viability assays established that ALI6 rescues the cells in a dose-dependent manner. When the cells were treated with ALI6 5 
minutes prior to adding Aß42, $1 \mu \mathrm{M}$ ALI6 reduces the cell death to $30 \%, 2 \mu \mathrm{M}$ ALI6

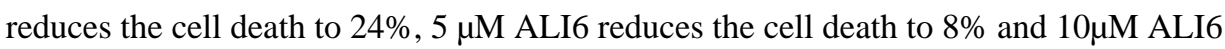

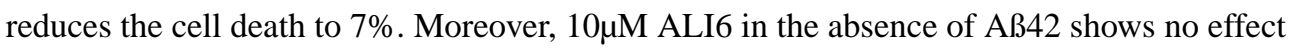
on cell viability. These results suggest that ALI6 inhibits Aß cytotoxicity.

\section{Validation of ALI6 with primary neurons}

Primary neuron models have been widely used to test $A B$ cytotoxicity and the effect of $A B$ inhibitors, and two known inhibitors of $A \beta$ curcumin $^{35}$ and (-)-epigallocatechin-3-gallate $(\mathrm{EGCG})^{36}$, have been reported to rescue the neurotoxic effects of $A ß$. We further validated the effect of ALI6 with mouse primary neurons. Cells from cortices dissected at embryonic day 15 were dispersed and cultured for 14 days in vitro (DIV14). Mouse cortical neurons were previously shown to express PirB at DIV14 ${ }^{37}$. Cells were then treated with $500 \mathrm{nM}$ FITC-Aß42 to assess Aß binding. We found that cells pre-treated with $10 \mu \mathrm{M}$ ALI6 bound $39.0 \pm 20.5 \%$ (mean \pm SD) of FITC-Aß42 compared to cells pre-treated with the same amount of DMSO (Fig. 6a \& b), indicating that ALI6 inhibits the binding of Aß to neurons. The observation that ALI6 does not fully inhibit A 3 binding, even at a higher dose $(50 \mu \mathrm{M}$ ALI6, bound $49.9 \pm 12.3 \%$ FITC-Aß42, Fig. $6 \mathrm{~b}$ ) indicates there are $A ß$ receptors other than LilrB2 on the neuronal cell surface, and is consistent with the observation of Aß42 binding to neuron cells from $\mathrm{PirB}^{-/-}$mice at $50 \%$ the level of wild-type neurons ${ }^{26}$.

Although the binding of $A B$ was not fully eliminated, we found that ALI6 is sufficient in inhibiting $A ß$ cytotoxicity in primary neurons similar to curcumin ${ }^{35}$ and $\mathrm{EGCG}^{36}$. Using terminal deoxynucleotidyl transferase-dUTP nick end labeling (TUNEL) assays to detect apoptotic DNA fragmentation, we found $50.1 \pm 4.3 \%$ of the cells treated with $\mathrm{A} B 42$ and DMSO undergo cell death (Fig. 6c \& d). When DMSO was substituted with same amount of ALI6 $(5 \mu \mathrm{M})$, cell death dropped to $16.5 \pm 8.9 \%$, equivalent to the vehicle control (11.9 $\pm 11.4 \%)$ and ALI6 alone $(11.0 \pm 11.6 \%)$. These results support the potential of ALI6 for rescuing $A ß$ caused neuron damage.

We further tested the effect of ALI6 on the downstream pathway of LilrB2. A previous study proposed that Aß-LilrB2 interaction causes dephosphorylation of cofilin, an actindepolymerizing factor, and leading to eventual synapse $\operatorname{loss}^{26}$. Indeed, the same study showed the phosphorylated cofilin (p-cofilin)/total cofilin level decreasing in primary

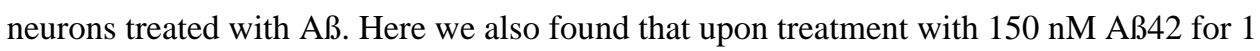
hour, the p-cofilin/cofilin level in primary neurons dropped to $67.5 \pm 8.1 \%$ the value seen in cells treated with vehicle alone (Fig. 6e). When pre-treated with $3 \mu \mathrm{M}$ ALI6, the p-cofilin/ cofilin level was restored to $101.5 \pm 7.4 \%$ relative to vehicle treated cells. These results indicate ALI6 protects neurons from Aß-induced changes in the cofilin signaling pathway, and further support the therapeutic potential of ALI6.

\section{DISCUSSION}

Our interaction assays confirm previous reports that LilrB2 recognizes $A ß$ oligomers $^{26}$, and suggest a molecular mechanism for the specificity of recognition. We first mapped the binding core to the segment ${ }^{16} \mathrm{KLVFFA}^{21}$ of $\mathrm{A} \beta$ and tested two binding epitopes: a tandem repeat of ${ }^{16} \mathrm{KLVFFA}^{21}\left(\mathrm{~A} \beta_{16-21}\right.$-TR) designed to spontaneously self-assemble into an anti- 


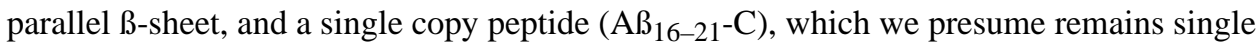
stranded in solution under the conditions tested due to an entropy barrier to oligomer formation. We found that LilrB2 binds to the tandem repeat but not to the single strand (Fig. 1), suggesting that LilrB2 recognizes an antiparallel $\beta$-sheet conformation specific to $A \beta$ oligomers $^{31}$.

Several lines of evidence support our hypothesis that $A \beta_{16-21}$-TR is a better mimic of the full-length $A \beta$ oligomer than is $A \beta_{16-21^{-}} \mathrm{C}$. $A \beta_{16-21}$-TR has more $B$ strand content than $A \beta_{16-21}-\mathrm{C}$ as indicated by a higher ellipticity $(C D)$ value measured at $200 \mathrm{~nm}$ for the peptides linked to the $5 \mathrm{x}$ arginine tag (this tag was needed to achieve sufficient solubility) (Supplementary Fig. 2, Supplementary Table 1). The analysis of CD spectra also shows that the $B$ strand (antiparallel) content of $A \beta_{16-21}$-TR is higher (33\%) than that of $A \beta_{16-21}-\mathrm{C}$ (28\%). Moreover, when incubated at $37^{\circ} \mathrm{C}$ at high concentration $(2 \mathrm{mM}), \mathrm{A} \beta_{16-21}-\mathrm{TR}$ formed fibers but $A \beta_{16-21}-\mathrm{C}$ did not (Supplementary Fig. 2). Presuming that fibrillar and oligomeric species share common structural features, these results suggest that $A \beta_{16-21}$-TR better mimics the full length $A ß$ oligomer and explain our observation that it is a better epitope for LilrB2. Finally, the Rosetta docking experiments using as input the steric zipper structure of KLVFFA successfully generated a model that agrees with our LilrB2benzamidine complex structure within the top $0.1 \%$ of Rosetta energy rankings. These results support our previous hypothesis that that $\beta$-sheets are not only characteristic of amyloid fibers ${ }^{38}$ but also of oligomers ${ }^{39,40}$.

The transient and heterogeneous nature of $A ß$ oligomers makes their structural elucidation extremely challenging. The observation that LilrB2 binds to $A ß$ oligomers with a wide range of sizes (Supplementary Fig. 2) also indicates the difficulty of characterizing the structure of an $\mathrm{A} B$ oligomer-LilrB2 complex. To gain insights into the structure of this complex, we first narrowed the binding core of $A \beta$ to a six-residue segment $\left({ }^{16} \mathrm{KLVFFA}^{21}\right)$, and identified its binding site on LilrB2 through the structure of LilrB2 with a small molecule that mimics phenylalanine sidechains of the $\mathrm{A} \beta$ binding core. The binding sites were validated by mutagenesis and Rosetta docking, and then used for structure-based inhibitor design. Our results show that the LilrB2 D1D2-benzamidine complex structure we determined provides a platform sufficient for inhibitor development of the Aß-LilrB2 interaction.

In addition to LilrB2, other putative $A ß$ receptors have been reported to bind $A B$ oligomers and cause neuronal damage ${ }^{25}$. Our rationale for choosing LilrB2 as a target for inhibitor design is that an animal model shows that mice lacking PirB (the murine homolog of LilrB2) are immune to the damaging effects of $A B$ in hippocampal long-term potentiation (LTP) and memory ${ }^{26}$. One reason to suppose that blocking only the LilrB2 receptor might be sufficient to inhibit $A ß$ toxicity is that blockade of one high-affinity $A ß$ receptor may sufficiently reduce the contact of $A B$ with cells. This hypothesis is supported by the observation that $\mathrm{A} \beta 42$ oligomer binding to cultured cortical neurons from $\mathrm{PirB}^{-1-}$ mice is diminished by about $50 \%$ relative to wild-type neurons ${ }^{26}$. In $\mathrm{AD}$ patients, this reduction may be sufficient to move the equilibrium from $A ß$-cell contact to $A ß$ clearance ${ }^{41}$, thus inhibiting $A ß$ triggered neuronal toxicity. Our cell viability assays on primary neurons support this hypothesis, which show ALI6 can almost completely block the effect of Aß (Fig. 6d). Further study is required to identify the possibility that our inhibitor can also work on other $A B$ receptors. 
Compared to other $\mathrm{AD}$ drug development strategies that target $\mathrm{A} ß$ aggregation (e.g. ${ }^{15-17}$ ) or bind monomeric $A ß$ with antibody ${ }^{42}$, targeting $A ß$ oligomer is advantageous because the inhibitor does not need to be added before $A ß$ aggregation. When testing the inhibition of $A ß$ cytotoxicity, $A ß$ monomer or aggregation targeting inhibitors need to be co-incubated with $A B$ from the beginning of $A ß$ aggregation (e.g. ${ }^{15}$ ); therefore, presumably these inhibitors can only treat early-stage $\mathrm{AD}$ patients before massive $\mathrm{A} \beta$ aggregation forms. This may be part of the reason why solanezumab, an antibody targeting monomeric $A B$ failed in recent clinical trial ${ }^{43}$. In comparison, all of our inhibition experiments were done by separately adding inhibitors and pre-formed $A ß$ oligomer, offering the possibility of treating patients that already have $A B$ aggregation in their brains.

Structure-based approach has been shown to be a powerful tool for drug development (e.g. ${ }^{5,44}$ ). In this study, we computationally identified 12 candidate inhibitors by structure-guided selection. Nine out of 12 candidates show inhibition of Aß-LilrB2 interaction in vitro; 6 candidates were selected for further testing, and all of them exhibit low micro molar to high nanomolar $\mathrm{K}_{\mathrm{i}}$ and $\mathrm{IC}_{50}$ values. These inhibitors eliminate the effects of Aß-LilrB2 binding on the cell surface, and candidate ALI6 inhibits Aß binding and cytotoxicity to primary neurons. Our results support the hypothesis that blocking this $A \beta$-receptor interaction is a potential way to inhibit $A ß$ toxicity and prevent neuron damage, and that LilrB2 is a promising therapeutic target. In addition, the compound library we used for computational inhibitor selection is composed of approved drugs, drugs in animal tests and clinical trials, and natural products whose pharmacokinetic (PK) and/or toxicity profile is known. During the inhibitor selection process, we also checked the potential of the selected compounds to cross the blood-brain barrier (BBB, Supplementary Table 3). This strategy ensures our selected inhibitors, e.g. ALI6, are safe for human use and are able to cross the BBB. These properties make these inhibitors useful tools in further investigation of the role of LilrB2 in pathogenesis of $\mathrm{AD}$ and qualifies them as promising candidates for expediting further $\mathrm{AD}$ drug development. We also note that concentrations of inhibitors we used are high and might be difficult to achieve in vivo. Our proof-of-concept study provides several promising starting points for drug development, and further work is needed to improve the affinity of these inhibitors to increase their translational values.

\section{METHODS AND MATERIALS}

Methods and materials used in this study are available in supplementary information.

\section{Supplementary Material}

Refer to Web version on PubMed Central for supplementary material.

\section{ACKNOWLEDGEMENTS}

We thank C. Shatz for providing the LilrB2 plasmid. This work is based upon research conducted at the Northeastern Collaborative Access Team beamlines, which are funded by the National Institute of General Medical Sciences from the National Institutes of Health (P41 GM103403). The Pilatus 6M detector on 24-ID-C beam line is funded by a NIH-ORIP HEI grant (S10 RR029205). This research used resources of the Advanced Photon Source, a U.S. Department of Energy (DOE) Office of Science User Facility operated for the DOE Office of Science by Argonne National Laboratory under Contract No. DE-AC02-06CH11357. We also thank NIH AG 054022 and 
DOE DE-FC02-02ER63421 for support. For requests of materials reported in this study, please contact David S. Eisenberg and Lin Jiang.

\section{Abbreviations:}

AB B-amyloid

AD Alzheimer's disease

LilrB2 D1D2 the D1 and D2 domains of LilrB2

Ben benzamidine

ALI Aß-LilrB2 interaction inhibitor

\section{REFERENCES}

1. Hardy JA \& Higgins GA Alzheimer's disease: the amyloid cascade hypothesis. Science 256, 184-5 (1992). [PubMed: 1566067]

2. Sheng M, Sabatini BL \& Sudhof TC Synapses and Alzheimer's disease. Cold Spring Harb Perspect Biol 4(2012).

3. Braak H \& Braak E Alzheimer's disease: striatal amyloid deposits and neurofibrillary changes. J Neuropathol Exp Neurol 49, 215-24 (1990). [PubMed: 1692337]

4. Nagy $\mathrm{Z}$ et al. Influence of the apolipoprotein E genotype on amyloid deposition and neurofibrillary tangle formation in Alzheimer's disease. Neuroscience 69, 757-61 (1995). [PubMed: 8596645]

5. Seidler PM et al. Structure-based inhibitors of tau aggregation. Nat Chem 10, 170-176 (2018). [PubMed: 29359764]

6. Tanzi RE \& Bertram L Twenty years of the Alzheimer's disease amyloid hypothesis: a genetic perspective. Cell 120, 545-55 (2005). [PubMed: 15734686]

7. Bettens K, Sleegers K \& Van Broeckhoven C Current status on Alzheimer disease molecular genetics: from past, to present, to future. Hum Mol Genet 19, R4-R11 (2010). [PubMed: 20388643]

8. Lambert MP et al. Diffusible, nonfibrillar ligands derived from Abeta1-42 are potent central nervous system neurotoxins. Proc Natl Acad Sci U S A 95, 6448-53 (1998). [PubMed: 9600986]

9. Lesne $\mathrm{S}$ et al. A specific amyloid-beta protein assembly in the brain impairs memory. Nature 440, 352-7 (2006). [PubMed: 16541076]

10. Li S et al. Soluble Abeta oligomers inhibit long-term potentiation through a mechanism involving excessive activation of extrasynaptic NR2B-containing NMDA receptors. J Neurosci 31, 6627-38 (2011). [PubMed: 21543591]

11. Hsiao K et al. Correlative memory deficits, Abeta elevation, and amyloid plaques in transgenic mice. Science 274, 99-102 (1996). [PubMed: 8810256]

12. Ashe KH \& Zahs KR Probing the biology of Alzheimer's disease in mice. Neuron 66, 631-45 (2010). [PubMed: 20547123]

13. Sorrentino $\mathrm{V}$ et al. Enhancing mitochondrial proteostasis reduces amyloid-beta proteotoxicity. Nature 552, 187-193 (2017). [PubMed: 29211722]

14. Zhu B et al. ER-associated degradation regulates Alzheimer's amyloid pathology and memory function by modulating gamma-secretase activity. Nat Commun 8, 1472 (2017). [PubMed: 29133892]

15. Cheng PN, Liu C, Zhao M, Eisenberg D \& Nowick JS Amyloid beta-sheet mimics that antagonize protein aggregation and reduce amyloid toxicity. Nat Chem 4, 927-33 (2012). [PubMed: 23089868]

16. Bradley Yat-Wah Man et al. Group 9 metal-based inhibitors of ß-amyloid (1-40) fibrillation as potential therapeutic agents for Alzheimer's disease. Chemical Science 2, 917 (2011).

17. Ehrnhoefer DE et al. EGCG redirects amyloidogenic polypeptides into unstructured, off-pathway oligomers. Nat Struct Mol Biol 15, 558-66 (2008). [PubMed: 18511942] 
18. Dovey HF et al. Functional gamma-secretase inhibitors reduce beta-amyloid peptide levels in brain. J Neurochem 76, 173-81 (2001). [PubMed: 11145990]

19. Evin G Future Therapeutics in Alzheimer's Disease: Development Status of BACE Inhibitors. BioDrugs 30, 173-94 (2016). [PubMed: 27023706]

20. Mitani Y et al. Differential effects between gamma-secretase inhibitors and modulators on cognitive function in amyloid precursor protein-transgenic and nontransgenic mice. J Neurosci 32, 2037-50 (2012). [PubMed: 22323718]

21. Castillo-Carranza DL, Guerrero-Munoz MJ \& Kayed R Immunotherapy for the treatment of Alzheimer's disease: amyloid-beta or tau, which is the right target? Immunotargets Ther 3, 19-28 (2014). [PubMed: 27471697]

22. Sevigny $\mathbf{J}$ et al. The antibody aducanumab reduces Abeta plaques in Alzheimer's disease. Nature 537, 50-6 (2016). [PubMed: 27582220]

23. Lauren J, Gimbel DA, Nygaard HB, Gilbert JW \& Strittmatter SM Cellular prion protein mediates impairment of synaptic plasticity by amyloid-beta oligomers. Nature 457, 1128-32 (2009). [PubMed: 19242475]

24. Cisse $\mathrm{M}$ et al. Reversing EphB2 depletion rescues cognitive functions in Alzheimer model. Nature 469, 47-52 (2011). [PubMed: 21113149]

25. Jarosz-Griffiths HH, Noble E, Rushworth JV \& Hooper NM Amyloid-beta Receptors: The Good, the Bad, and the Prion Protein. J Biol Chem 291, 3174-83 (2016). [PubMed: 26719327]

26. Kim $\mathrm{T}$ et al. Human LilrB2 is a beta-amyloid receptor and its murine homolog PirB regulates synaptic plasticity in an Alzheimer's model. Science 341, 1399-404 (2013). [PubMed: 24052308]

27. Luhrs T et al. 3D structure of Alzheimer's amyloid-beta(1-42) fibrils. Proc Natl Acad Sci U S A 102, 17342-7 (2005). [PubMed: 16293696]

28. Colletier JP et al. Molecular basis for amyloid-beta polymorphism. Proc Natl Acad Sci U S A 108, 16938-43 (2011). [PubMed: 21949245]

29. Willcox BE et al. Crystal structure of LIR-2 (ILT4) at 1.8 A: differences from LIR-1 (ILT2) in regions implicated in the binding of the Human Cytomegalovirus class I MHC homolog UL18. BMC Struct Biol 2, 6 (2002). [PubMed: 12390682]

30. Raveh B, London N, Zimmerman L \& Schueler-Furman O Rosetta FlexPepDock ab-initio: simultaneous folding, docking and refinement of peptides onto their receptors. PLoS One 6, e18934 (2011). [PubMed: 21572516]

31. Stroud JC, Liu C, Teng PK \& Eisenberg D Toxic fibrillar oligomers of amyloid-beta have crossbeta structure. Proc Natl Acad Sci U S A 109, 7717-22 (2012). [PubMed: 22547798]

32. Kuhlman B \& Baker D Native protein sequences are close to optimal for their structures. Proc Natl Acad Sci U S A 97, 10383-8 (2000). [PubMed: 10984534]

33. Lawrence MC \& Colman PM Shape complementarity at protein/protein interfaces. J Mol Biol 234, 946-50 (1993). [PubMed: 8263940]

34. Jiang L et al. Structure-based discovery of fiber-binding compounds that reduce the cytotoxicity of amyloid beta. Elife 2, e00857 (2013). [PubMed: 23878726]

35. Huang HC, Chang P, Dai XL \& Jiang ZF Protective effects of curcumin on amyloid-beta-induced neuronal oxidative damage. Neurochem Res 37, 1584-97 (2012). [PubMed: 22476982]

36. He Y et al. Prolonged exposure of cortical neurons to oligomeric amyloid-beta impairs NMDA receptor function via NADPH oxidase-mediated ROS production: protective effect of green tea (-)-epigallocatechin-3-gallate. ASN Neuro 3, e00050 (2011). [PubMed: 21434871]

37. Syken J, Grandpre T, Kanold PO \& Shatz CJ PirB restricts ocular-dominance plasticity in visual cortex. Science 313, 1795-800 (2006). [PubMed: 16917027]

38. Gremer L et al. Fibril structure of amyloid-beta(1-42) by cryo-electron microscopy. Science 358, 116-119 (2017). [PubMed: 28882996]

39. Liu $\mathrm{C}$ et al. Out-of-register beta-sheets suggest a pathway to toxic amyloid aggregates. Proc Natl Acad Sci U S A 109, 20913-8 (2012). [PubMed: 23213214]

40. Laganowsky A et al. Atomic view of a toxic amyloid small oligomer. Science 335, 1228-31 (2012). [PubMed: 22403391] 
41. Nalivaeva NN, Belyaev ND, Kerridge C \& Turner AJ Amyloid-clearing proteins and their epigenetic regulation as a therapeutic target in Alzheimer's disease. Front Aging Neurosci 6, 235 (2014). [PubMed: 25278875]

42. Crespi GA, Hermans SJ, Parker MW \& Miles LA Molecular basis for mid-region amyloid-beta capture by leading Alzheimer's disease immunotherapies. Sci Rep 5, 9649 (2015). [PubMed: 25880481]

43. Abbott A \& Dolgin E Failed Alzheimer's trial does not kill leading theory of disease. Nature 540, 15-16 (2016). [PubMed: 27905452]

44. Wang S et al. D4 dopamine receptor high-resolution structures enable the discovery of selective agonists. Science 358, 381-386 (2017). [PubMed: 29051383] 


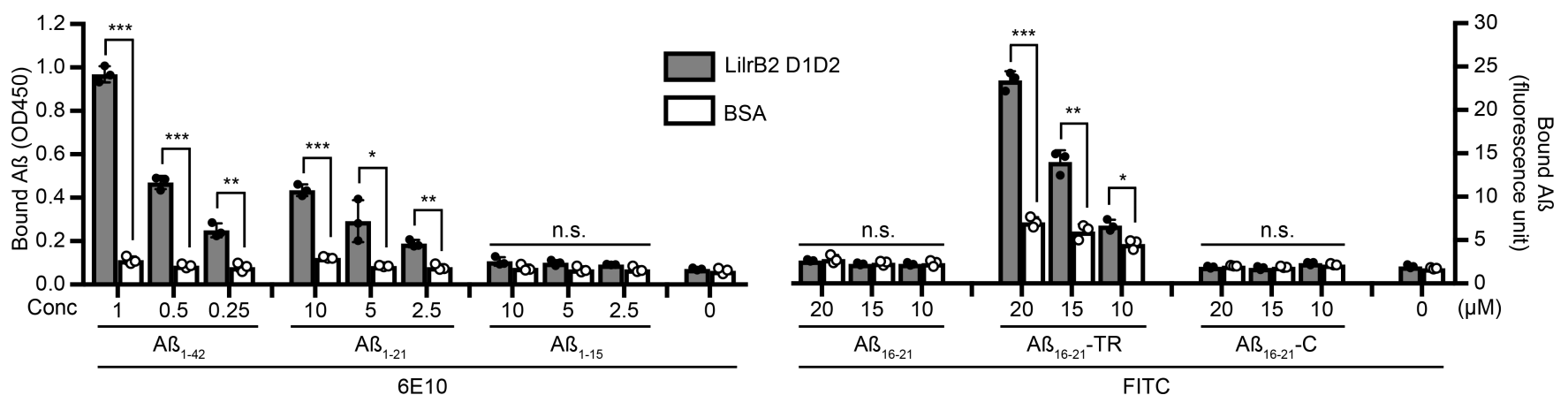

Figure 1. The ${ }^{16}$ KLVFFA21 segment of AB binds to LilrB2 D1D2.

ELISA-based interaction assays of Aß42 and its constituent segments. LilrB2 D1D2 (black bars) or bovine serum albumin as a negative control (BSA, white bars) was immobilized on ELISA plates, and incubated with $A ß$ segments at concentrations shown. The unbound segments were washed off and the amounts of bound $A ß$ segments were measured by the $A B$ specific antibody $6 \mathrm{E} 10$ and quantified by absorbance at wavelength $450 \mathrm{~nm}$ (OD450, left panel), or measured by the fluorescence signal of fluorescein (FITC) conjugated to the amino-termini of the segments and quantified by fluorescence units (right panel). Notice that for $A \beta_{1-42}, A \beta_{1-21}$ and $A \beta_{16-21}-T R$, the amounts of $A \beta_{\text {segments bound to LilrB2 D1D2 }}$ were significantly higher than that to BSA, indicating interaction between these segments and LilrB2 D1D2. The absence of KLVFFA from the weak binder $A \beta_{1-15}$, as well as its presence in the stronger binders $A \beta_{1-21}$, and $A \beta_{16-21}$-TR (sequence

KLVFFAPDGKLVFFA), indicate ${ }^{16} \mathrm{KLVFFA}^{21}$ is the key segment of $A \beta$ that binds to LilrB2. Segment sequences are shown in Supplementary Table 1. Data are means \pm SD ( $n=3$ independent experiments). Two-sided $t$ tests were performed and detailed statistical analyses are reported in Supplementary Table 4. *, p<0.05; **, $\mathrm{p}<0.005 ; * * *, p<0.0005$, n.s., not significant; conc, concentration. 
a

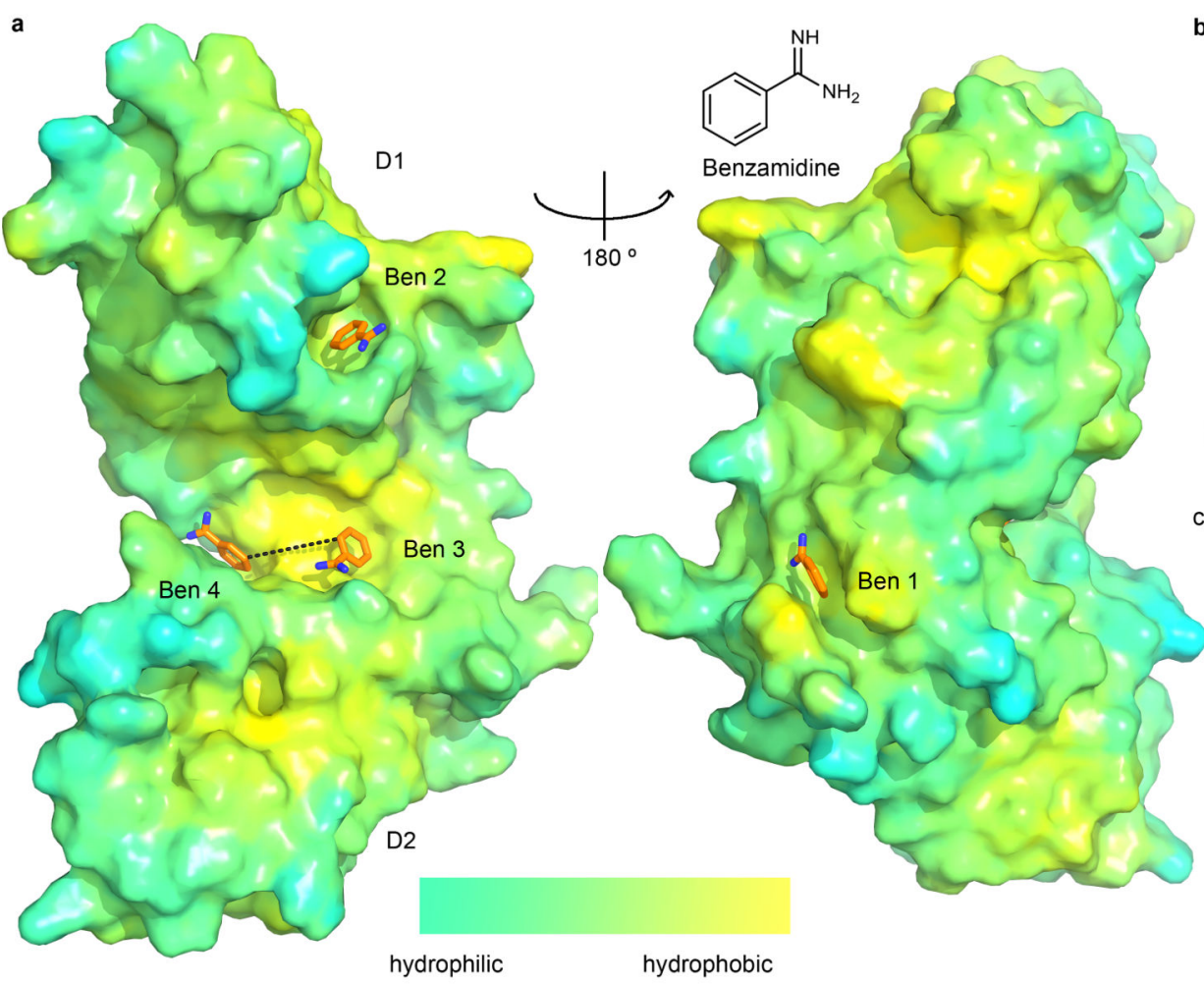

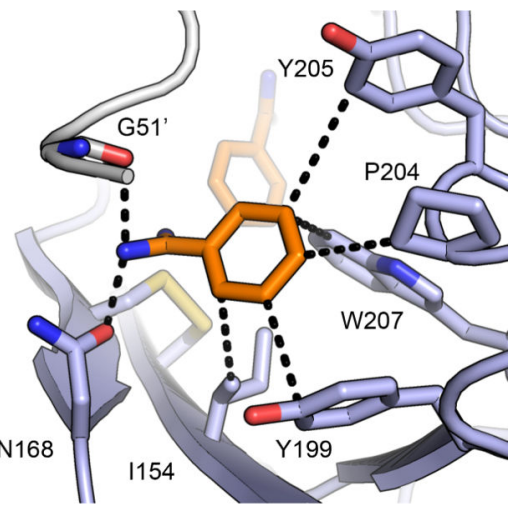

C

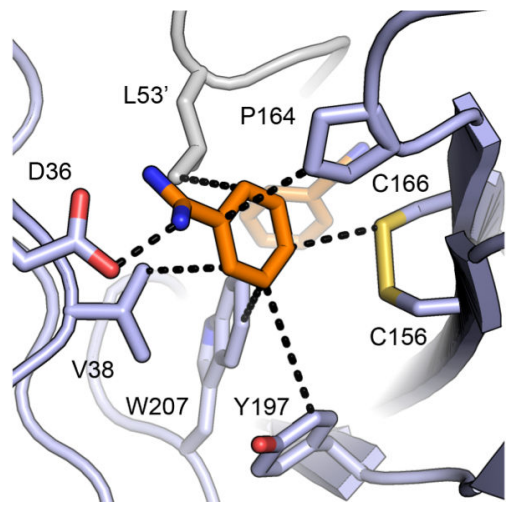

Figure 2. Crystal structure of LilrB2 D1D2 complexed with benzamidine.

a, Overview of the structure of LilrB2 D1D2 (shown in surface model, colored by hydrophobicity) complexed with benzamidine (Ben 1 to 4, shown in sticks). The chemical structure of benzamidine is shown at the top right corner. The black dashed line between Ben 3 and Ben 4 represents $7.5 \AA$ A. Hydrophobicity ranges from -1.7 (hydrophilic) to +3.7 (hydrophobic). Notice that the binding pockets of Ben 3 and Ben 4 are located at the groove between LilrB2 domains D1 and D2, and the groove has an extended hydrophobic surface. $\mathbf{b}$ and $\mathbf{c}$, detailed interaction of Ben 3 (b) and Ben 4 (c) with LilrB2. LilrB2 is shown as a cartoon and the side chains of the residues involved in benzamidine binding are shown as sticks. The black dashed lines represent distances between $2.4 \AA$ and $4.8 \AA$. 

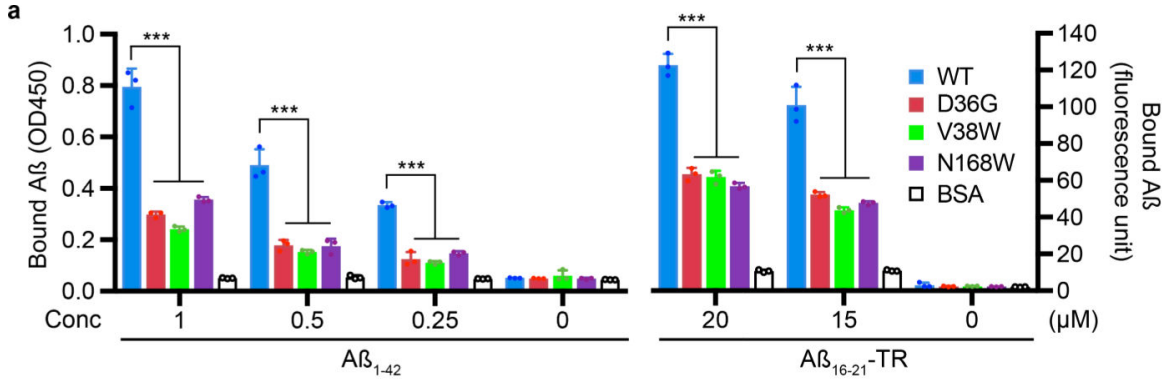

b

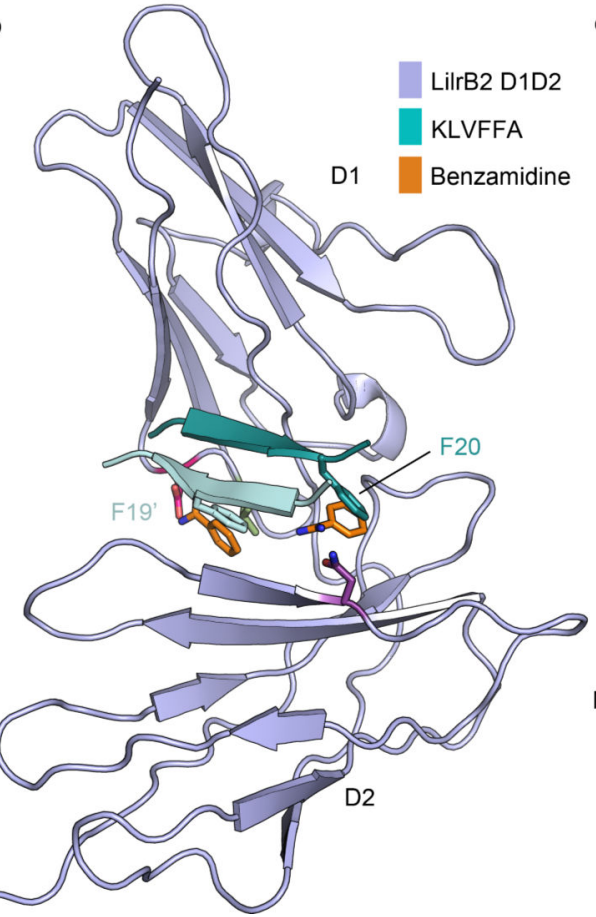

c

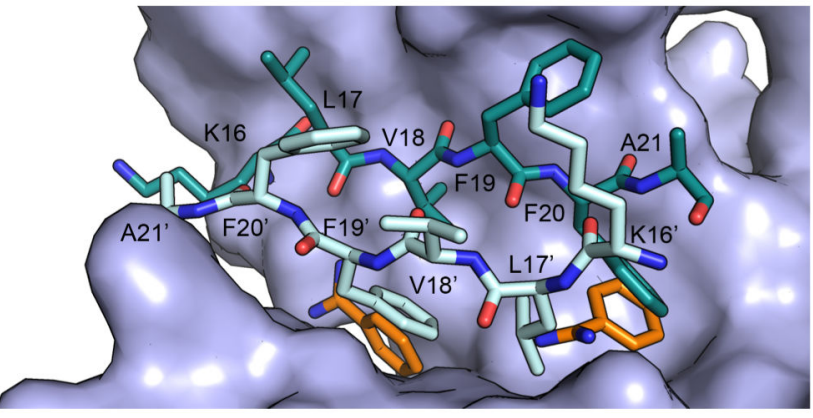

Figure 3. Mutagenesis studies and Rosetta docking validate the AB binding sites on LilrB2. a, ELISA-based interaction assays using wild type LilrB2 D1D2 (WT) or designed mutants. The same amount of LilrB2 D1D2 WT (blue bars), D36G (red bars), V38W (green bars) and N168W (purple bars), as well as bovine serum albumin (BSA, white bars) was immobilized on an ELISA plate (loading control see Supplementary Fig. 4), and incubated with A $\beta_{1-42}$ or $\mathrm{A} \beta_{16-21}-\mathrm{TR}$ at indicated concentrations. The amounts of bound $\mathrm{A} \beta_{1-42}$ were measured by antibody 6E10 and quantified by absorbance at wavelength $450 \mathrm{~nm}$ (OD450, left panel); the amounts of bound $A \beta_{16-21}$-TR were measured by fluorescence signal of fluorescein and quantified by fluorescence units (right panel). Data are means \pm SD ( $n=3$ independent experiments, $* * * \mathrm{p}<0.0005$, ANOVA test); conc, concentration. For detailed statistical analysis see Supplementary Table 4. b-d, Model of two KLVFFA peptides binding to LilrB2 D1D2 calculated by Rosetta docking. In this model Phe ${ }^{20}$ from one KLVFFA chain and Phe ${ }^{19}$ ' from another chain bind to Ben 3 (d, left panel) and Ben 4 (d, right panel) pockets respectively. Three residues tested in mutagenesis studies $\left(\mathrm{Asp}^{36}, \mathrm{Val}^{38}\right.$ and $\mathrm{Asn}{ }^{168}$ ) were used as restraints in Rosetta docking. Residue colors correspond to the key given in panel (a). 
a

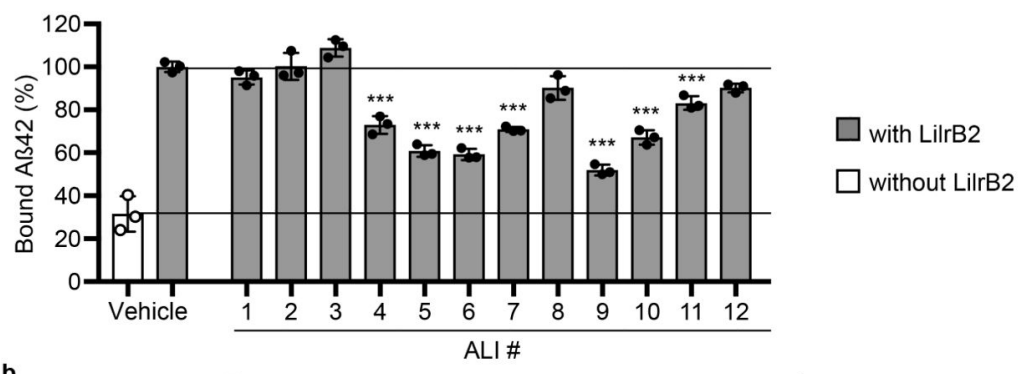

b
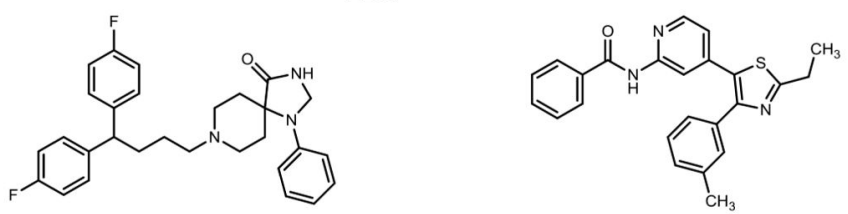

ALI10: TAK-715
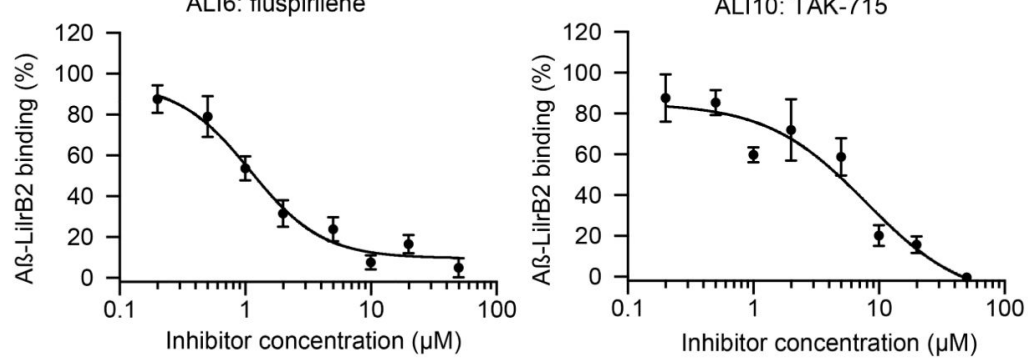

d

\begin{tabular}{clcc}
\hline ALI $\#$ & \multicolumn{1}{c}{ Name } & $\mathbf{K}_{\mathrm{i}}(\boldsymbol{\mu M})$ & $\mathbf{I C}_{50}(\boldsymbol{\mu M})$ \\
\hline 4 & Miconazole & $1.51 \pm 0.11$ & $5.67 \pm 0.61$ \\
5 & Sertaconazole nitrate & $2.92 \pm 0.67$ & $18.1 \pm 1.54$ \\
6 & Fluspirilene & $0.32 \pm 0.07$ & $1.09 \pm 0.52$ \\
7 & CDPPB & $3.57 \pm 0.58$ & $12.7 \pm 1.32$ \\
9 & ML169 & $0.89 \pm 0.16$ & $2.98 \pm 0.16$ \\
10 & TAK-715 & $1.01 \pm 0.16$ & $7.79 \pm 0.78$ \\
\hline
\end{tabular}

c
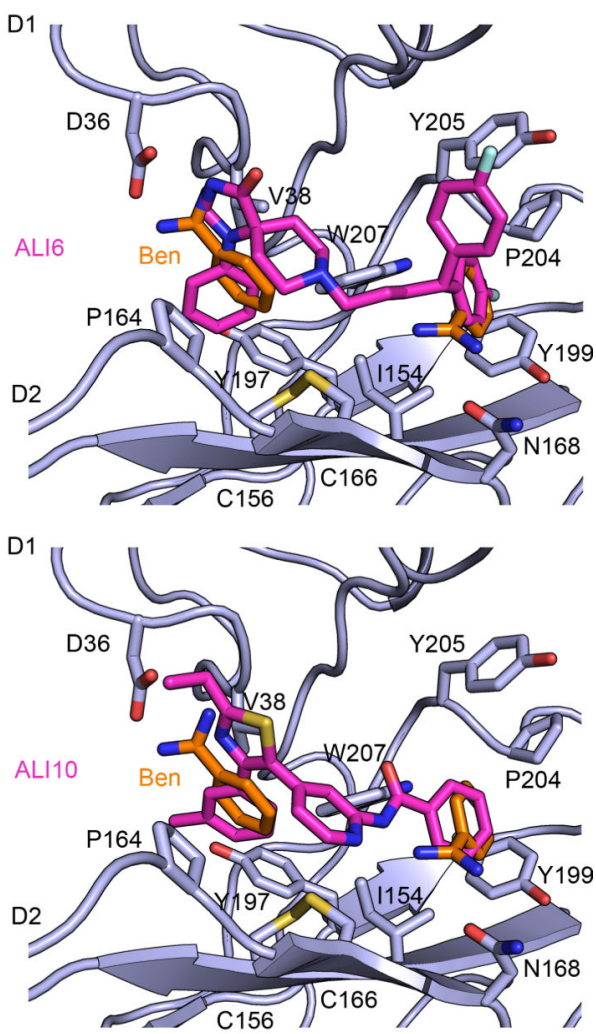

Figure 4. Selected small molecules inhibit the AB-LilrB2 interaction in vitro.

a, immunoprecipitation assays of $\mathrm{A} \beta 42$ with (black bars) or without (white bar) LilrB2

D1D2. $1 \mu \mathrm{M}$ of $\mathrm{A} 342$ and $100 \mathrm{nM}$ of LilrB2 D1D2 were mixed with $5 \mu \mathrm{M}$ of AB-LilrB2 inhibitors (ALI \#1-12) or equal amounts of DMSO (vehicle) and the amount of bound Aß42 was quantified by ELISA. Data are presented as percentages relative to controls in which LilrB2 and vehicle was added. Data are means \pm SD $(n=3$ independent experiments, $* * * \mathrm{p}<0.0005$, ANOVA test). For detailed statistical analysis see Supplementary Table 4. b, same immunoprecipitation assays using multiple concentrations of ALI6 (left) and ALI10 (right). ELISA absorbance values of samples without LilrB2 were subtracted as a background from those of samples with LilrB2. The data are presented as percentages relative to the samples with LilrB2 and vehicle. The percentage values of samples with inhibitors are plotted against the concentration of inhibitors. The name and chemical structure of inhibitors is shown on the top of each panel. c, Docking models of ALI6 (upper panel) and ALI10 (lower panel) binding to Ben 3 and 4 pockets. Residues involved in benzamidine binding are shown as stick models. $\mathbf{d}, \mathrm{K}_{\mathrm{i}}$ and $\mathrm{IC}_{50}$ values calculated from the 
data are shown in (b) and Supplementary Fig. 6. In immunoprecipitation assays shown in (b) and (d), data are mean $\pm \mathrm{SD}, \mathrm{n}=3$ independent experiments. 


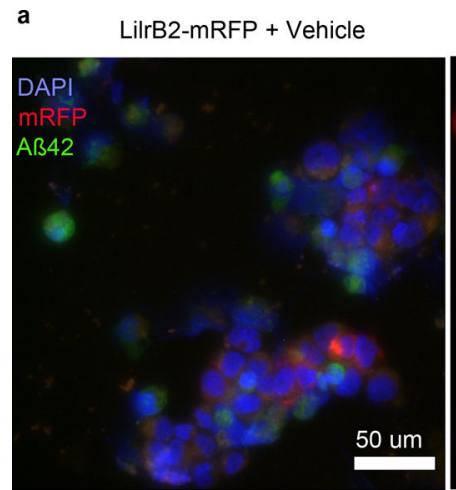

LilrB2-mRFP + ALI6
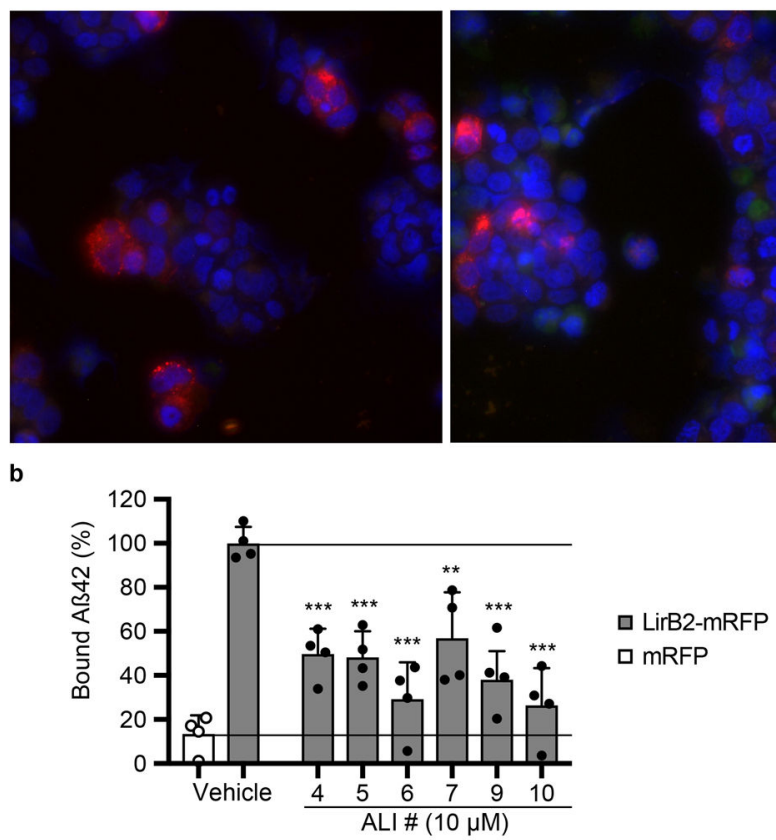

mRFP + Vehicle

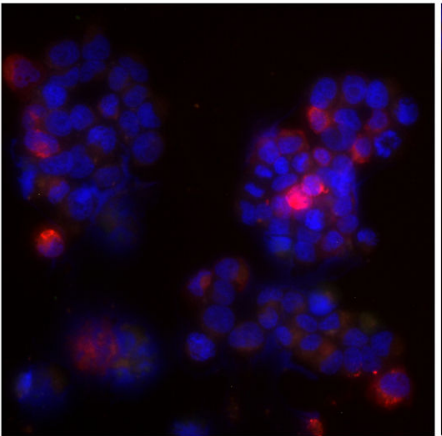

LilrB2-mRFP + ALI7

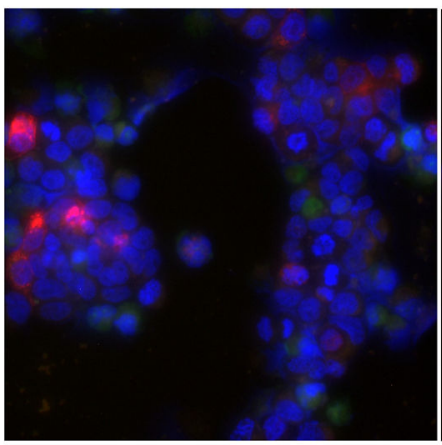

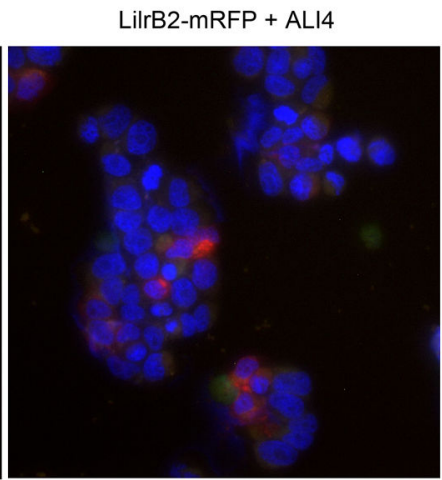

LilrB2-mRFP + ALI9
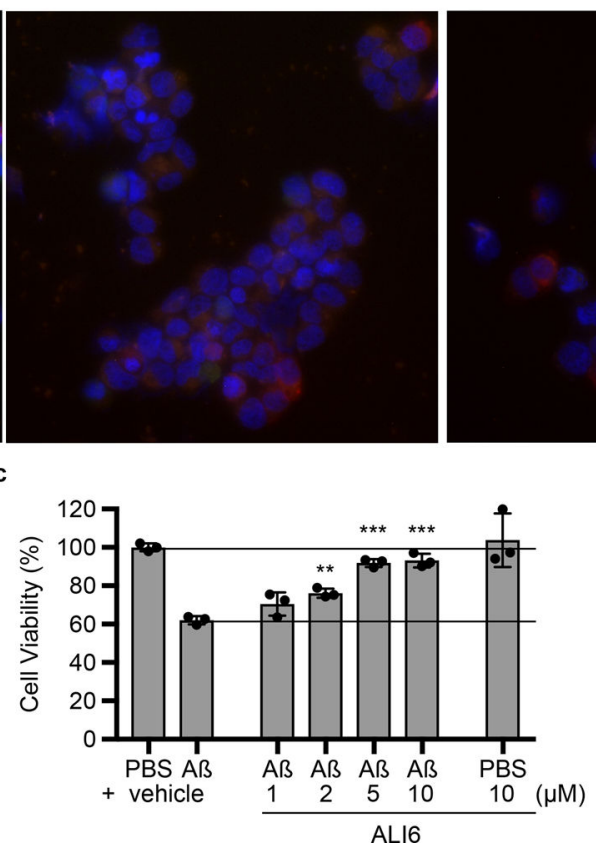

LilrB2-mRFP + ALI5

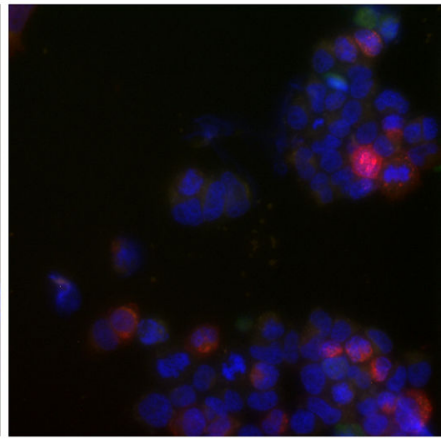

LilrB2-mRFP + ALI10

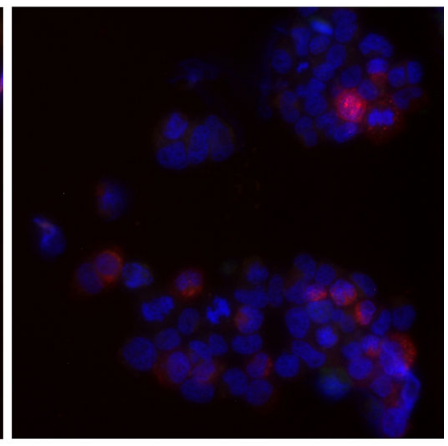

Figure 5. Selected inhibitors block LilrB2 induced cell attachment and inhibit toxicity of AB. a, Fluorescent images of HEK293T cells transfected with LilrB2-mRFP or mRFP (red), and treated with $500 \mathrm{nM}$ fluorescein conjugated to AB42 (FITC-Aß, green) and $10 \mu \mathrm{M}$ selected Aß-LilrB2 inhibitors (or equal amounts of DMSO as vehicle control). DAPI, 4', 6diamidino-2-phenylindole. b, Quantification of FITC-A342 binding represented in (a). Aß42 binding was quantified as integrated intensity of green fluorescence in each well, normalized to LilrB2 expression level quantified as integrated intensity of red fluorescence in the same well (or normalized to cell confluency for cells transfect with mRFP), and then presented as a percentage relative to the controls, which are LilrB2-mRFP transfected HEK293T cells treated with vehicle. Data are means \pm SD ( $n=4$ independent experiments, $* * p<0.005$, $* * * \mathrm{p}<0.0005$, ANOVA test). c, Cell viability (MTT) assays show that ALI6 reduces the toxicity of Aß42. HEK293T cells transfected with LilrB2-mRFP were treated with indicated concentrations of ALI6 or vehicle control, and then $500 \mathrm{nM}$ of oligomeric Aß42 or PBS control was added. Cell viability is shown as a percentage relative to controls in which only PBS and vehicle are added. Data are means \pm SD ( $n=3$ independent experiments, $* * p<0.005$, $* * * \mathrm{p}<0.0005$, ANOVA test). For detailed statistical analysis see Supplementary Table 4. 
$a$

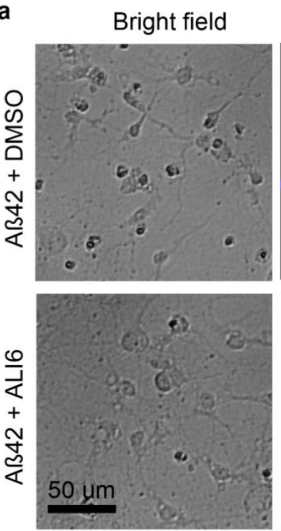

c
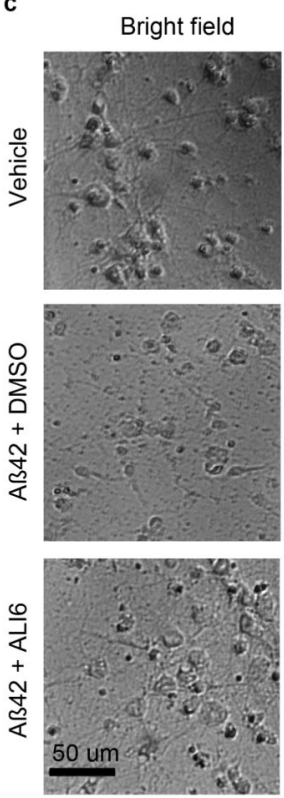

Hoechst
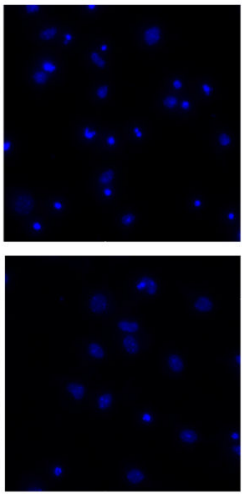

Hoechst
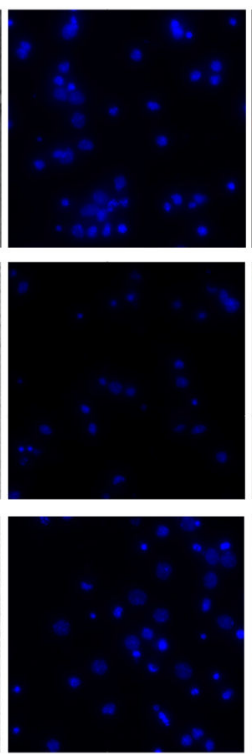
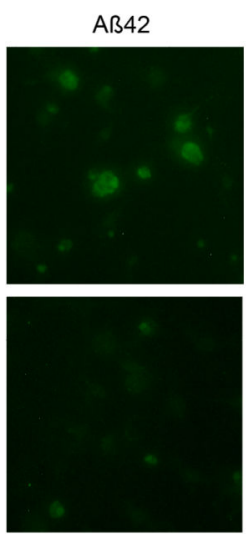

TUNEL
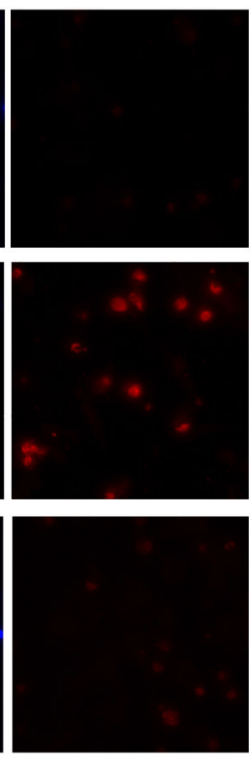
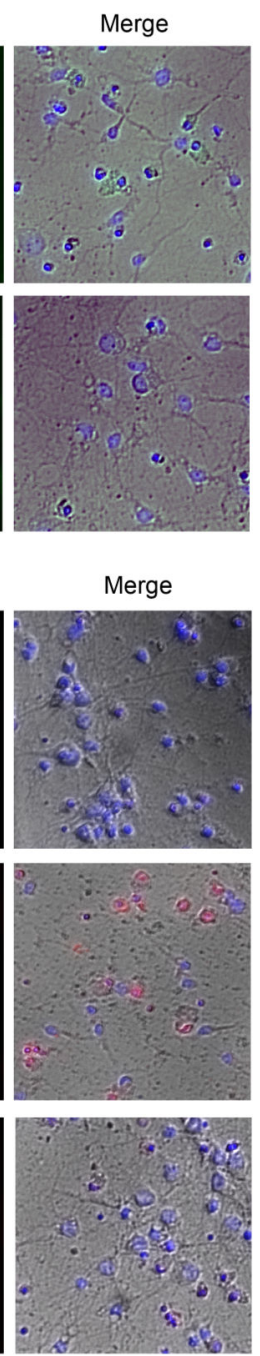
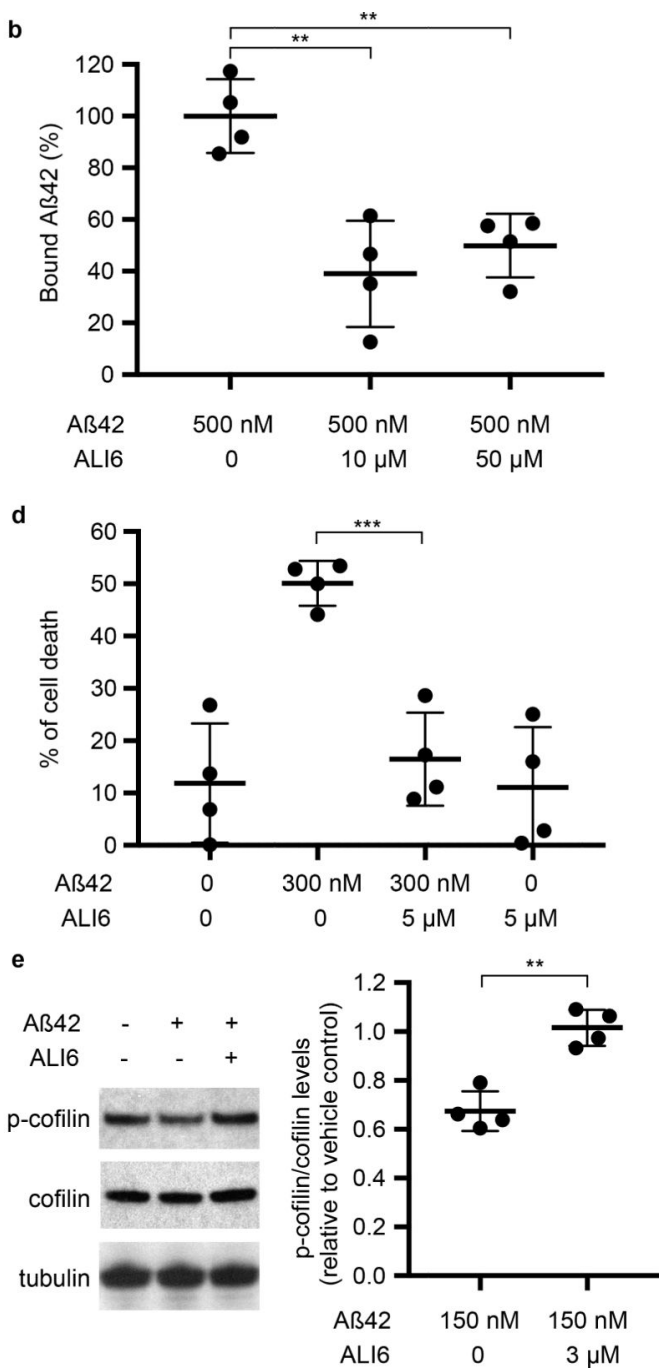

Figure 6. Validation of ALI6 using primary neurons.

a, Bright field and fluorescence images of primary neurons treated with $500 \mathrm{nM}$ FITC-A 3 (green) and $10 \mu \mathrm{M}$ ALI6 (or equal amounts of DMSO). b, Quantification of FITC-Aß42 binding represented in (a). Aß42 binding was quantified as integrated intensity of green fluorescence in each well, normalized to cell confluency in the same well, and then presented as a percentage relative to cells treated with FITC-Aß42 and DMSO $(* * p<0.005$, ANOVA test). c, Bright field and fluorescence images of primary neuron cells treated with $300 \mathrm{nM}$ Aß42 and $5 \mu \mathrm{M}$ ALI6 or equal amounts of DMSO, or treated with PBS and DMSO as vehicle control. Cell viability was measured by TUNEL assays and dead cells are shown as red puncta. d, Quantification of TUNEL cell viability assays. Cell viability is shown as a percentage of cell death calculated as the number of red puncta divided by the number of blue puncta (Hoechst stain) $(* * * \mathrm{p}<0.0005$, two-sided $\mathrm{t}$ test). e, Primary neuron cells were treated with $150 \mathrm{nM}$ Aß42 with $3 \mu \mathrm{M}$ ALI6 or equal amounts of DMSO, and cofilin signaling levels were analyzed by Western blotting (left). Anti-Tubulin $\beta-3$ antibody detects neuronal tubulin and was used as a loading control. Quantification of cofilin phosphorylation (right) was calculated as the intensity of phosphorylated cofilin band divided by the intensity 
of cofilin band, and was normalized to the cells treated with PBS and DMSO (vehicle control) $(* * \mathrm{p}<0.005$, two-sided $\mathrm{t}$ test). All Data are means $\pm \mathrm{SD}(\mathrm{n}=4$ independent experiments). For detailed statistical analysis see Supplementary Table 4. 